\title{
Diagnosis and treatment of nerve injury following venipuncture - A report of two cases -
}

\section{In Jung Kim ${ }^{1}$, Hana Cho ${ }^{1}$, Myung Sub Yi ${ }^{1}$, Yong Hee Park ${ }^{1,2}$, Sujin $\mathrm{Kim}^{3}$, Duk Kyung Kim${ }^{4}$, and Hwa-Yong Shin ${ }^{1,2}$}

'Department of Anesthesiology and Pain Medicine, Chung-Ang University Hospital, ${ }^{2}$ Department of Anesthesiology and Pain Medicine, Chung-Ang University College of Medicine, ${ }^{3}$ Department of Radiology, Chung-Ang University Hospital, Chung-Ang University College of Medicine, ${ }^{4}$ Department of Anesthesiology and Pain Medicine, Samsung Medical Center, School of Medicine, Sungkyunkwan University, Seoul, Korea

Background: Venipuncture is one of the one of the most commonly performed, minimally-invasive procedures; however, it may lead to peripheral nerve injury. Here, we describe the diagnosis, treatment, and prognosis of two self-reported cases of nerve injury during venipuncture with the aim of drawing attention to possible needle-related nerve injuries.

Case: Two anesthesiologists in our hospital experienced an injury of the lateral antebrachial cutaneous branch of the musculocutaneous nerve during venipuncture. Immediately, they underwent ultrasound examinations and nerve blocks with oral medication, resulting in full recovery.

Conclusions: Ultrasonography is important for the early and confirmative diagnosis of a nerve injury during venipuncture, and for immediate treatment with a nerve block. Moreover, it is imperative for both the practitioner and the patient to be aware of the possible complication of nerve injury after venipuncture.

Keywords: Anesthesiologists; Peripheral nerve injuries; Ultrasonography; Venipuncture.

\section{Corresponding author}

Hwa-Yong Shin, M.D., Ph.D., FIPP, CIPS

Department of Anesthesiology and

Pain Medicine, Chung-Ang University

College of Medicine, 102 Heukseok-

ro, Dongjak-gu, Seoul 06973, Korea

Tel: 82-2-6299-3164

Fax: 82-2-6299-2585

E-mail: pain@cau.ac.kr
Venipuncture, or phlebotomy, is one of the most commonly performed minimally-invasive procedures, essential for routine blood sampling, intravenous fluid therapy, transfusion, and blood donation. Although this procedure is relatively harmless in most cases, it may be associated with a critical peripheral nerve injury such as complexion regional pain syndrome (CRPS) [1,2].

A vein in the antecubital fossa is one of the first choices for routine blood sampling in adults. Specifically, the median cubital vein, located between the cephalic and basilic vein, is a large vein suitable for blood sampling. Other veins commonly used for blood sampling that are also located in the antecubital fossa include the cephalic vein, basilic vein, and median antebrachial vein [3]. However, the antecubital fossa is an anatomically complex area, in which the neurovascular structures may lie very close to each other, making the nerves vulnerable to injury during venipuncture. Moreover, the anatomical variations in the antecubital fossa between individuals add to the risk of nerve injury [4].

The incidence of nerve injury during venipuncture varies in different reports, ranging from $1 / 21,000-1 / 25,000$ to as low as $1 / 67,000[2,5,6]$. While most of these patients fully recover, a rare possibility exists that they may experience severe, chronic pain that met the diagnostic criteria of CRPS.

This is an Open Access article distributed under the terms of the Creative Commons Attribution Non-Commercial License (http://creativecommons.org/licenses/by-nc/4.0) which permits unrestricted non-commercial use, distribution, and reproduction in any medium, provided the original work is properly cited.

Copyright (c) the Korean Society of Anesthesiologists, 2021 
Here, we present two self-reported cases of anesthesiologists experiencing nerve injury during blood sampling. Written consent for publication was obtained from the patients. We also discuss the importance of ultrasonography for the early diagnosis and prompt treatment of nerve injury following venipuncture.

\section{CASE REPORTS}

\section{Case 1}

A 28-year-old male patient undergoing venous blood sampling was punctured in the middle of the antecubital fossa of the left arm with a 21-gauge needle. During the procedure, he felt a sharp, electrical pain throughout his arm, extending from the venipuncture site to the tip of the fingers. The intensity of the pain was rated as 8/10 in the visual analog scale (VAS). After the needle was withdrawn, he experienced dysesthesia and burning pain in the anterior wrist and the anterior, lower half of his forearm, which was rated as 7-8/10 on the VAS and lasted for more than $24 \mathrm{~h}$.

Since the pain and discomfort did not subside even after $24 \mathrm{~h}$, he visited the pain clinic for evaluation and management. After a brief medical interview and physical examination to rule out peripheral neuropathy, ultrasonographic examination of the venipuncture site was performed to accurately assess the nerve. It revealed segmental swelling and perineural echogenic changes in the lateral antebrachial cutaneous branch of the musculocutaneous nerve at the fore- arm level (Fig. 1A), between the biceps brachii tendon and the punctured cephalic vein at the antecubital level, compared with contralateral side, suggestive of neuritis with perineural hemorrhage (Fig. 1B).

Low amplitudes in the left lateral and medial antebrachial cutaneous nerves were observed on a sensory nerve conduction study (NCS) performed on the same day, suggesting left lateral and medial antebrachial cutaneous neuropathy.

Consequently, a nerve block of the injured nerve was promptly performed on the same day. After local infiltration with $2 \%$ mepivacaine, a mixture of $0.75 \%$ ropivacaine $(1 \mathrm{ml})$ , triamcinolone (20 mg) , and normal saline $(2.5 \mathrm{ml})$ was injected around the nerve (Fig. 2). The patient experienced

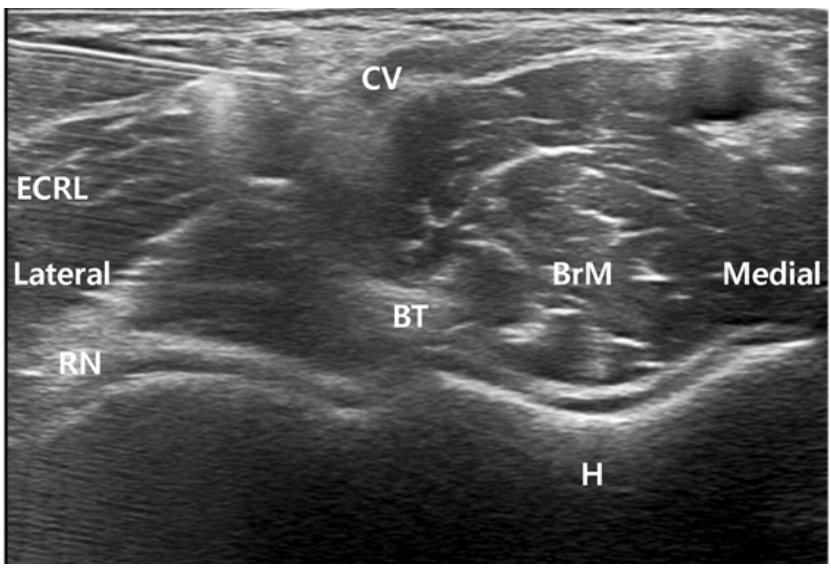

Fig. 2. Ultrasound guided peripheral nerve block for the lateral antebrachial cutaneous nerve at the elbow. BrM: brachialis muscle, BT: biceps tendon, CV: cephalic vein, ECRL: extensor carpi radialis longus muscle, $\mathrm{H}$ : humerus, $\mathrm{RN}$ : radial nerve.
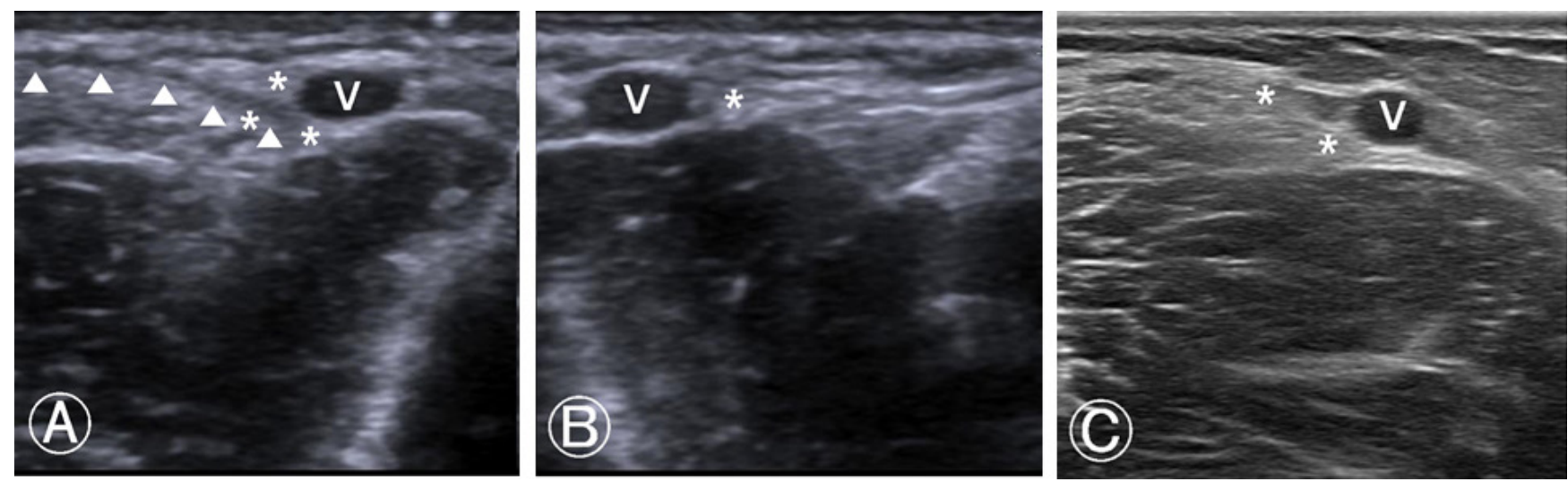

Fig. 1. Ultrasonography at the antecubital level. (A) Left, injured arm; it shows the soft tissue swelling and prominent echogenicity around the lateral antebrachial cutaneous nerve (asterisks), adjacent to the cephalic vein (V), implying either direct nerve injury or compressive neuritis due to the surrounding hematoma. A hyoechoic linear line indicated with ' $\mathbf{A}$ ' symbol shows the trajectory of the needle coming out. (B) A contralateral, right, uninjured arm; it shows the lateral antebrachial cutaneous nerve (asterisk) with normal sono-archtitecture. (C) Follow-up ultrasonographic image of the first case done at three weeks after the injury, showing improvement in the segmental swelling and perineural echogenic changes around the affected nerve (asterisks). 
immediate pain relief, with no adverse effect. After the procedure, the patient reported a pain intensity of $1 / 10$ on the VAS. Additionally, he was prescribed with prednisolone (5 $\mathrm{mg}$ ), pregabalin (75 mg), tramadol (75 mg), acetaminophen (650 mg), and esomeprazole (20 mg) per os bis in die for a week.

The patient experienced an intermittent shooting, electrical pain (2-3/10 in the VAS) extending anteriorly from his forearm to his wrist for a week after administering the nerve block. The medications, including pregabalin (75 mg), naproxen (500 mg), and esomeprazole (20 mg) bis in die per os were prescribed for another week. In the following two weeks after the injury, his pain and discomfort gradually reduced to a degree that was ignorable.

A follow-up ultrasonographic examination at three weeks after the injury showed an improvement in the segmental swelling and perineural echogenic changes around the affected nerve, suggesting an improvement in the neuritis and perineural hemorrhage (Fig. 1C). The patient no longer experienced pain, discomfort, or paresthesia.

\section{Case 2}

A 32-year-old female patient was punctured with a 21-gauge needle in the middle of the antecubital fossa of the left arm for venous blood sampling. She felt an electrical sensation from her forearm to the tip of her fingers for a few seconds when her skin was punctured, followed by numbness after the needle was removed. Subsequently, she complained of a dysesthesia extending from her elbow to the tips of her first four fingers, and dull pain rated 7-8/10 on the VAS around the punctured area. She visited the pain clinic that afternoon.

Ultrasonographic examination of the antecubital area was performed to further evaluate the puncture site. Its results suggested probable injury of the lateral antebrachial cutaneous nerve, which lies adjacent to the median cubital vein, with mild surrounding infiltrates, as compared with the contralateral side. It showed similar ultrasonographic findings with Fig. 1A, B in Case 1.

After the ultrasonographic examination of the nerve injury, skin infiltration with $2 \%$ mepivacaine was performed and a nerve block of the injured nerve was performed with a mixture of $0.75 \%$ ropivacaine $(1 \mathrm{ml})$, triamcinolone $(20 \mathrm{mg})$, and normal saline $(2.5 \mathrm{ml})$ was injected around the nerve. Immediately after the nerve block, her pain reduced to $1-2 / 10$ on the VAS, with hypesthesia in the affected forearm.
She was prescribed prednisolone $(5 \mathrm{mg})$, pregabalin (75 $\mathrm{mg}$ ), tramadol (37.5 mg), acetaminophen ( $325 \mathrm{mg}$ ), and esomeprazole $(20 \mathrm{mg})$ per os bis in die for a week.

The pain around the puncture site worsened (6-7/10 in the VAS) $24 \mathrm{~h}$ after the nerve block, with further worsening through the following day (8/10 in the VAS). Following the administration of oral medication, the pain gradually subsided to $1-2 / 10$ on the VAS over the course of a week.

The patient continued to experience intermittent shooting pain at the puncture site during arm movement, but without any sensory deficit. The oral medications, including pregabalin (75 mg), naproxen (500 mg), and esomeprazole (20 mg) per os bis in die were prescribed for another week. She had complete pain relief at 2 weeks after the injury without any tingling sensation and numbness.

Three and a half weeks later, a follow-up ultrasonographic examination was performed. Its results revealed a decreased extent of the echogenic lesion adjacent to the nerve in the left arm, which had been noted in the previous examination (similar findings with Fig. 1C). She was pain free, both at rest and with arm and hand movement.

\section{DISCUSSION}

This study is a report of two anesthesiologists' self-reported cases of venipuncture-related nerve injury. They were able to describe the detailed clinical features of the condition from the doctor's and the patient's perspective throughout the course of the injury.

First and foremost, it is important to prevent nerve injury during venipuncture by carefully selecting the puncture site with the least risk of nerve injury. A typical venipuncture site is chosen among superficial veins in the antecubital fossa of the forearm. The lateral cutaneous nerve is seen deep along the cephalic vein in most cases. Risk of nerve damage is higher especially when the cephalic vein is punctured in the lower part of the cubital fossa, making it a dangerous area for venipuncture. The medial cutaneous nerve descends medial to the basilic vein, either passing over or under the ulnar portion of the median cubital vein. These anatomical relationships between veins and cutaneous nerves in the antebrachial fossa suggest that the radial side of median cubital vein is a relatively safe site for venipuncture $[7,8]$. Even with the safest puncture site, delicate maneuvering and careful handling of the needle or catheter is essential to avoid deep penetration, risking injuries of the nerve lying deep behind the vein. Additionally, multiple attempts in the 
same site should be avoided.

Despite an appropriate and satisfactory venipuncture, a possibility of a nerve injury still exists. When venipuncture-related nerve injury is suspected, one should first elicit a history of recent venipuncture through history taking and physical examination, and rule out any underlying diseases that might cause peripheral neuropathy, such as diabetes. For a more accurate and objective diagnosis, other tools can be used, including electromyography (EMG), NCS, magnetic resonance imaging (MRI) and ultrasonography.

EMG and NCS are useful to localize and confirm peripheral nerve injuries [9]. Decreased conduction velocity or conduction block in the affected nerve on NCS is also indicative of nerve injury. However, electrodiagnostic studies cannot provide detailed information regarding the anatomy of the injured area or determine the severity of the injury, and the patients experience major discomfort during these studies.

MRI can also aid in assessing nerve injuries. It is helpful in directly evaluating the changes in the nerve signals and perineural tissues, especially in the deeper structures that are hard to evaluate through ultrasound [9]. However, its expensive and time-consuming nature make MRI a less attractive diagnostic tool. In addition, MRI is contraindicated for some patients, including uncooperative children, patients with mechanical devices such as a pacemaker, or patients admitted to the intensive care unit.

Ultrasonography can be useful for localizing and diagnosing iatrogenic peripheral nerve injuries. Additionally, it can be used to assess the surrounding structures and detect abnormalities, such as hematoma. Doppler imaging can be used to assess the blood vessels and vascular changes in the area surrounding the injured nerve. Bedside ultrasonography is useful in the real-time assessment of nerve injuries, and can be performed in an acute phase of the injury without causing any pain to the patient, making it one of the most preferred tools for diagnosing peripheral nerve injury. Injection treatment can also be performed simultaneously with the diagnosis [10].

The first step in diagnosing an iatrogenic peripheral nerve injury through ultrasonography is identifying the lesion. A normal peripheral nerve is sheathed with an outer epineurium, which contains several fascicles composed of axons. A transducer should be placed at the nerve of interest at a 90-degree angle, and a cross sectional view of a normal peripheral nerve will show hyper-echoic epineurium with a few hypo-echoic fascicles within the epineurium. The pe- ripheral nerve is further differentiated from the surrounding muscles and tendons, which are relatively more hypoechoic.

Once the nerve of interest is identified in a cross-sectional view, the probe should be moved along the nerve to scan the nerve throughout its course. Ttracing the nerve of interest enables identifying any abnormalities in size and appearance, or discontinuation of the nerve. Turning the probe 90-degrees around can show a longitudinal image of the lesion. Longitudinal images can show changes in the nerve diameters or discontinuation of the nerves.

Ultrasonographic findings of a nerve injury include enlargement of nerve diameter indicating nerve swelling or neuroma, focal disorganization of its fascicular structure, or transection of the nerve. In a first-degree Sunderland nerve injury, or neurapraxia, the nerve retains its normal appearance with or without minor swelling of the nerve. A second-degree Sunderland nerve injury, or axonotmesis, shows a clearly enlarged diameter of the nerve due to axonal swelling and edema. Focal swelling and the surrounding edema can be shown with hyper-echogenicity. A third-degree or fourth-degree Sunderland nerve injury, or neurotmesis, shows loss of normal fascicular pattern due to involvement of the endoneurium. Hypo-echogenicity and marked enlargement of the nerve can also be noted. A fifth-degree Sunderland nerve injury is a complete transection of the nerve. In this kind of transection injury, ultrasound can detect discontinuation of the nerve, showing interruption of the epineurium and enlarged, hypo-echoic nerve stump. Distance between the lesion site, the affected muscles, and the skin can be measured and can help guide decision-making in treatment course.

Ultrasound scanning of the lesion site can not only detect abnormalities of the nerve itself, but also reveal abnormalities in the surrounding areas. Such findings include scars, foreign bodies, or hematoma. Sharp scars associated with laceration with a knife or glass, needle or catheter related injury can be seen as hypoechoic, linear lines penetrating the skin, subcutaneous tissues, or interrupting nerve sheaths. Foreign bodies can be usually found as scattering soundwaves from their surfaces. A hematoma can be seen as a diffuse, hypo-echoic area on the image.

Diagnosis of iatrogenic nerve injury and identification of the lesion site should lead to prompt treatment. The initial general management of nerve injury is similar to that of other nerve lesions. Conservative management includes the administration of oral medications, including opioids, NSAIDs, adjuvant analgesics, such as gabapentin or pregabalin [11], 
and systemic glucocorticoids, along with physiotherapy to prevent atrophy of the muscles supplied by the affected nerve.

Additionally, immediate nerve blockade upon diagnosis of a nerve injury is crucial. A nerve block using local anesthetics is both a diagnostic and therapeutic tool for peripheral nerve injury. Nerve block can result in immediate pain relief of the affected area, which can confirm the diagnosis of a nerve injury and the location of the lesion site. Ultrasonography also serves as a superior tool for the peripheral nerve block [12].

With the probe in the exact lesion site, the practitioner should carefully puncture the skin near the affected area. The needle tip should be visible while the needle is advanced, until the tip is placed desirably around the injured nerve. Pre-measured local anesthetics are injected through the needle and infiltrate around the injured nerve, which can be observed in real-time with the help of ultrasonography. Because the course of the needle is visible at all times, it is possible for the practitioner to avoid other nearby structures. Thus, the use of ultrasonography results in fewer complications with a shorter performance time. Local glucocorticoid injection is also used to inhibit the release of local inflammatory mediators and decrease ectopic neuronal discharges [13], aiding in a quicker recovery time.

Needle or catheter related nerve injury may also occur during the administration of regional anesthesia, such as brachial plexus block [14], or under general anesthesia. Central venous catheterization is among one of the most commonly performed catheterization procedures under general anesthesia. The incidence of nerve injury during subclavian central venous catheterization has been reported as $0.6 \%$ [15]. Such catheter related nerve injuries during procedures under regional or general anesthesia can be prevented by the routine application of ultrasound during these procedures. The practitioner can avoid nerve injuries by carefully inspecting the vein that is to be cannulated and its surrounding structures.

In conclusion, it is crucial to avoid nerve injury during venipuncture or catheter related procedures in the first place by carefully selecting the puncture site as well as the using ultrasonography for procedures with a risk of nerve injury. Furthermore, it is important for both the practitioner and the patient to be aware of the possibility of nerve injury during seemingly harmless procedures, such as venipuncture for routine blood sampling. When a peripheral nerve injury is suspected despite many efforts, application of ultra- sonography for the early detection and prompt diagnosis of nerve injury during needle or catheter related procedures as well as immediate nerve block of the affected nerve are of utmost importance in alleviating pain and shortening the course of the injury.

\section{CONFLICTS OF INTEREST}

No potential conflict of interest relevant to this article was reported.

\section{DATA AVAILABILITY STATEMENT}

All data generated or analyzed during this study are included in this published article. This is a case report.

\section{AUTHOR CONTRIBUTIONS}

Conceptualization: Hwa-Yong Shin. Visualization: Sujin Kim. Writing - original draft: In Jung Kim. Writing - review \& editing: Hana Cho, Myung Sub Yi, Yong Hee Park. Supervision: Hwa-Yong Shin, Duk Kyung Kim.

\section{ORCID}

In Jung Kim, https://orcid.org/0000-0002-3576-7987

Hana Cho, https://orcid.org/0000-0002-9077-9972

Myung Sub Yi, https://orcid.org/0000-0002-3527-0508

Yong Hee Park, https://orcid.org/0000-0001-6105-7383

Sujin Kim, https://orcid.org/0000-0003-2011-0752

Duk Kyung Kim, https://orcid.org/0000-0002-6555-2100

Hwa-Yong Shin, https://orcid.org/0000-0002-8721-3070

\section{REFERENCES}

1. Horowitz SH. Peripheral nerve injury and causalgia secondary to routine venipuncture. Neurology 1994; 44: 962-4.

2. Newman BH, Waxman DA. Blood donation-related neurologic needle injury: evaluation of 2 years' worth of data from a large blood center. Transfusion 1996; 36: 213-5.

3. Ialongo C, Bernardini S. Phlebotomy, a bridge between laboratory and patient. Biochem Med (Zagreb) 2016; 26: 17-33.

4. Kim HJ, Park SK, Park SH. Upper limb nerve injuries caused by intramuscular injection or routine venipuncture. Anesth Pain Med 2017; 12: 103-10.

5. Berry PR, Wallis WE. Venepuncture nerve injuries. Lancet 1977; 309: 1236-7. 
6. Tsukuda Y, Funakoshi T, Nasuhara Y, Nagano Y, Shimizu C, Iwasaki N. Venipuncture nerve injuries in the upper extremity from more than 1 million procedures. J Patient Saf 2019; 15: 299-301.

7. Yamada K, Yamada K, Katsuda I, Hida T. Cubital fossa venipuncture sites based on anatomical variations and relationships of cutaneous veins and nerves. Clin Anat 2008; 21: $307-$ 13.

8. Mikuni Y, Chiba S, Tonosaki Y. Topographical anatomy of superficial veins, cutaneous nerves, and arteries at venipuncture sites in the cubital fossa. Anat Sci Int 2013; 88: 46-57.

9. Fuller G. How to get the most out of nerve conduction studies and electromyography. J Neurol Neurosurg Psychiatry 2005; 76(Suppl 2): ii41-6.

10. Kim S, Choi JY, Huh YM, Song HT, Lee SA, Kim SM, et al. Role of magnetic resonance imaging in entrapment and compressive neuropathy--what, where, and how to see the peripheral nerves on the musculoskeletal magnetic resonance image: part
2. Upper extremity. Eur Radiol 2007; 17: 509-22.

11. Singh RK, Sinha VP, Pal US, Yadav SC, Singh MK. Pregabalin in post traumatic neuropathic pain: case studies. Natl J Maxillofac Surg 2012; 3: 91-5.

12. Lewis SR, Price A, Walker KJ, McGrattan K, Smith AF. Ultrasound guidance for upper and lower limb blocks. Cochrane Database Syst Rev 2015; 9: CD006459.

13. Eker HE, Cok OY, Aribogan A, Arslan G. Management of neuropathic pain with methylprednisolone at the site of nerve injury. Pain Med 2012; 13: 443-51

14. Kim HJ, Park SH, Shin HY, Choi YS. Brachial plexus injury as a complication after nerve block or vessel puncture. Korean J Pain 2014; 27: 210-8.

15. Ryan JA Jr, Abel RM, Abbott WM, Hopkins CC, Chesney TM, Colley R, et al. Catheter complications in total parenteral nutrition. A prospective study of 200 consecutive patients. N Engl J Med 1974; 290: 757-61. 